\title{
A AVALIAÇÃO DA ACUIDADE VISUAL DO ESCOLAR NO PROGRAMA SAÚDE NA ESCOLA: PROPOSTA DE VÍDEO PARA ORIENTAÇÃO DOCENTE
}

\section{ASSESSMENT OF VISUAL ACUITY IN THE SCHOOL HEALTH PROGRAM: VIDEO FOR TEACHERS GUIDANCE}

\author{
Mariana da Silva Braga ${ }^{1}$, Denise Celeste Godoy de Andrade Rodrigues ${ }^{1,2}$ \\ ${ }^{1}$ Centro Universitário de Volta Redonda (UniFOA)/ MECSMA/mbragaenf@ hotmail.com \\ ${ }^{2}$ Universidade do estado do Rio de Janeiro (UERJ)/ Faculdade de Tecnologia/ denisegodoy@uerj.br
}

\begin{abstract}
RESUMO
Este estudo buscou avaliar a acuidade visual de 166 alunos do $1^{\circ}$ ao $5^{\circ}$ ano da Escola Municipal Manoel Fonseca, no município de Barra do Piraí-RJ, de acordo com a tabela de Snellen, e verificar a percepção dos professores sobre a importância da avaliação da acuidade visual do escolar no Programa Saúde na Escola. Empregou-se questionário com questões abertas para a realização da coleta dos dados junto aos professores. Como resultados, 145 alunos $(87,3 \%)$ apresentaram acuidade visual normal e 21 alunos $(12,7 \%)$ apresentaram algum grau de déficit de acuidade visual. Aqueles que apresentaram déficit de acuidade visual foram encaminhados à Secretaria de Saúde do Município, para consulta. Este estudo resultou em um vídeo educativo, cujo título é "De Olho na Saúde", elaborado com o objetivo de ser um recurso didático para uso de docentes, visando à capacitação dos mesmos para a realização de testes de acuidade visual junto aos alunos.
\end{abstract}

Palavras-chave: Programa Saúde na Escola; Acuidade visual; Capacitação em serviço; Educação em Saúde; Enfermagem.

\begin{abstract}
This study evaluated the visual acuity of 166 students from 1st to 5th grade Municipal School Manuel Fonseca, in Barra do Pirai, Rio de Janeiro, according to the Snellen chart, and checks the teachers' perception of the importance of evaluating the visual acuity of the school in School Health Program. This is a descriptive study with a qualitative approach, which was used a questionnaire with open questions for the realization of data collection with teachers. The results show that 145 students $(87.3 \%)$ had normal visual acuity and 21 students $(12.7 \%)$ had some degree of visual acuity deficit. Those who showed deficits in visual acuity were referred to the Health Department of the Municipality for an eye appointment. This study resulted in an educational video, titled "Eye Health", developed with the goal of being a teaching resource for use by teachers, aimed at training them to perform visual acuity tests with student's network public elementary school in the city.
\end{abstract}

Key-words: School Health Program; Visual acuity; School; Inservice training, Health Education, Nursing. 


\title{
INTRODUÇÃO
}

Ao abordar a questão da promoção e prevenção em saúde, torna-se indispensável esclarecer que a promoção da saúde é mais ampla e abrangente, pois esta última procura identificar e enfrentar os determinantes do processo de saúde-doença, buscando transformá-los favoravelmente na direção da saúde. Já a prevenção das doenças busca que os indivíduos fiquem isentos das mesmas (SISTON; VARGAS, 2007).

Siston e Vargas (2007, p. 5), ao abordarem a relação entre promoção e prevenção da saúde escolar, esclarecem que:

\begin{abstract}
A promoção da saúde escolar busca modificar condições de vida para que sejam dignas e adequadas; aponta para a transformação dos processos de tomada de decisão dos escolares para que sejam predominantemente favoráveis à qualidade de vida e à saúde; e orienta-se ao conjunto de ações e decisões coletivas da comunidade escolar que possam favorecer a saúde e a melhoria das condições de bem estar.

Já a prevenção, orienta-se mais às ações de detecção, controle e enfraquecimento dos fatores de risco ou fatores causais de grupos de enfermidades ou de uma enfermidade específica. Seu foco é a doença e os mecanismos para atacá-la mediante o impacto sobre os fatores mais íntimos que a geram ou precipitam.
\end{abstract}

É notório o papel do profissional de enfermagem na prevenção de agravos à saúde da população. No desenvolvimento de programas de prevenção e controle de alterações da saúde, incluindo-se as oculares, o enfermeiro é elemento essencial na prevenção, pois com sua atuação em exames de triagem com escolares ele é capaz de detectar agravos à saúde, principalmente as alterações oculares, e exercer sua função de educador em saúde. Em exames de triagem da acuidade visual nas escolas cabe a este profissional informar sobre os principais sinais e sintomas de alterações oculares, mostrar aos pais a importância do encaminhamento do seu filho ao especialista, quando este apresenta alguma evidência de déficit ocular, e, ainda, capacitar os professores para realizar o teste com a escala de Snellen (DANTAS et al., 2009).

Assim sendo, ao discursar sobre promoção da saúde, mostra que o enfermeiro pode e deve atuar através de atividades de educação para a saúde e de práticas educativas, em diferentes espaços, como por exemplo, nas escolas. Nesse sentido, temse a educação para saúde como um processo que visa à promoção de saúde de escolares.

Estudos mostram que em relação à avaliação da acuidade visual, ela vem sendo realizada nas escolas por meio do Teste de Snellen, utilizando-se o professor como examinador. No entanto, segundo Gasparetto et al. (2004), esta é uma situação que tem sido motivo de polêmica, gerando descrédito em relação à realização do teste pelos Edição Especial com os melhores trabalhos apresentados no IV ENECiências: UFF - 13 a 16 de maio de 2014. 


\section{Ensino, Saúde e Ambiente - V 7 (1), Edição Especial, maio de 2014}

professores. Em estudo realizado em escolas públicas de Campinas-SP, os autores verificaram que os professores apresentaram pouco conhecimento sobre a saúde ocular e sugerem a implantação de um programa de saúde ocular em todo o sistema público de ensino, visando desenvolver ações de prevenção da incapacidade visual, promoção e recuperação da saúde ocular (GASPARETTO, 2004).

Diante do exposto, este trabalho teve por o objetivo geral de investigar a prevalência de doenças oculares em crianças e adolescentes matriculados em uma escola da rede municipal do município de Barra do Piraí, estado do Rio de Janeiro, através do teste de acuidade visual. Como objetivos específicos, realizar o teste de acuidade visual com os alunos; verificar qual a percepção dos professores sobre a importância da prevenção da saúde visual dos alunos; produzir um vídeo educativo para docentes sobre os métodos para aferir a acuidade visual de aluno do Ensino Fundamental.

\section{METODOLOGIA}

O estudo teve como cenário a Escola Municipal Manoel Fonseca, instituição de ensino situada em Barra do Piraí - RJ. Essa escola aderiu ao Programa Saúde na Escola (PSE) somente em 2012, apesar de o Programa ter sido instituído em 2007, com abrangência nacional.

Participaram do estudo 30 professores da Escola Municipal Manoel Fonseca que estavam atuando no ano de 2012. A população discente estudada foi constituída de 166 alunos matriculados no primeiro segmento do Ensino Fundamental no ano de 2012.

O estudo foi realizado com a prévia autorização da Secretaria Municipal de Educação e do diretor da Escola Municipal Manoel Fonseca, sendo aprovado pelo Comitê de Ética em Pesquisa do Centro Universitário de Volta Redonda (COEP/UniFOA) sob n ${ }^{\circ}$ CAAE- 05453112700005237.

Para a coleta de dados junto aos docentes utilizou-se como ferramenta um questionário com perguntas abertas. A acuidade visual dos alunos foi medida através da Escala Optométrica de Snellen, por profissional de enfermagem, em uma sala cedida pela direção da escola, em condições de iluminação e espaço adequados para o propósito da pesquisa. 
Ensino, Saúde e Ambiente - V 7 (1), Edição Especial, maio de 2014

\section{RESULTADOS E DISCUSSÃO}

Da população de 194 alunos pertencentes aos turnos da manhã e tarde, 28 alunos não participaram do estudo pelo fato de seus responsáveis não terem assinado o termo de consentimento, correspondendo a 14,4\%, deixando uma amostra válida de 166 alunos $(85,6 \%)$. Deste total, 91 pertenciam ao sexo feminino e 75 ao masculino. Cabe ressaltar que 10 alunos faziam uso de óculos e foram avaliados com estes recursos.

A idade dos escolares variou de 6 a 15 anos, sendo que 68,1\% tinham idade entre 9 e 12 anos. A Tabela 1 apresenta a distribuição por faixa etária e sexo.

Tabela 1: Distribuição dos alunos por faixa etária e sexo.

\begin{tabular}{|c|c|c|c|c|c|}
\hline \multirow[b]{2}{*}{ Etária } & \multirow[b]{2}{*}{ Faixa } & \multicolumn{3}{|c|}{$\mathbf{N}^{\mathbf{0}}$ de Alunos } & \multirow[b]{2}{*}{$\%$} \\
\hline & & $\begin{array}{ll} & \text { Masc } \\
\text { ulino } & \\
\end{array}$ & ino Femin & Soma & \\
\hline 08 anos & $06 \quad \mathrm{a}$ & 13 & 30 & 43 & 25,9 \\
\hline 12 anos & $\begin{array}{ll}09 & \mathrm{a} \\
\end{array}$ & 55 & 58 & 113 & 68,1 \\
\hline 15 anos & $13 \quad \mathrm{a}$ & 07 & 03 & 10 & 6,0 \\
\hline & Soma & 75 & 91 & 166 & 100,0 \\
\hline
\end{tabular}

Entre a amostragem válida dos escolares, 145 alunos (87,3\%) apresentaram acuidade visual normal e 21 alunos $(12,7 \%)$ apresentaram algum grau de déficit de acuidade visual. No olho direito 158 alunos $(95,2 \%)$ apresentaram acuidade visual normal e 8 alunos $(4,8 \%)$ apresentaram baixa acuidade visual. No olho esquerdo 153 alunos $(92,2 \%)$ apresentaram acuidade visual normal e 13 alunos $(7,8 \%)$ apresentaram baixa acuidade visual.

Estes resultados são mais elevados do que aqueles encontrados por Laignier, Castro e Sá (2010) em uma escola municipal de Vitória-ES, que, ao aplicarem o Teste de Snellen numa amostra de 168 crianças, apenas 6\% apresentaram baixa acuidade visual. Como também dos encontrados por Fendi (2008) em escolares da rede municipal de Marília-SP, participantes do programa "Olho no olho", em que o resultado foi $8,8 \%$.

Por outro lado, ficou bem abaixo dos resultados encontrados por Santa Ritta (2005), em estudo realizado em Florianópolis, onde o percentual de alunos com déficit da acuidade visual alcançou 19,7\% de uma amostra de 476 crianças. Assim como também por Granzoto et al. (2003), na avaliação de alunos do $1^{\circ}$ ano do ensino Edição Especial com os melhores trabalhos apresentados no IV ENECiências: UFF - 13 a 16 de maio de 2014. 


\section{Ensino, Saúde e Ambiente - V 7 (1), Edição Especial, maio de 2014}

fundamental pertencentes a 21 escolas municipais de Pelotas-RS, cujos resultados foram de $15,1 \%$. Couto Júnior et al. (2007) observaram em seus estudos que cerca de $15 \%$ das crianças da primeira série escolar têm alguma alteração visual e somente $20 \%$ delas faz acompanhamento assistencial de saúde.

Frente aos resultados encontrados pelo presente estudo, decidiu-se por encaminhar para a Secretaria de Saúde do Município os 21 alunos que apresentaram déficit da acuidade visual, para que fossem submetidos à consulta oftalmológica com especialista. Dos 8 alunos com baixa acuidade visual do olho direito, 2 alunos do sexo masculino foram encaminhados para consulta prioritária e 6 alunos (3 do sexo masculino e 3 do sexo feminino) para consulta regular. Dos 13 alunos com baixa acuidade visual no olho esquerdo, 4 alunos ( 3 do sexo masculino e 1 do sexo feminino) foram encaminhados para consulta prioritária e 9 alunos ( 4 do sexo masculino e 5 dos sexo feminino) para consulta regular.

Dos 21 alunos encaminhados para consulta, verificou-se que 10 alunos $(47,6 \%)$ têm a idade de 10 anos, sendo 1 do sexo feminino e 9 do sexo masculino. Em quatro alunos $(19,0 \%)$ foi diagnosticado déficit bilateral de acuidade visual.

Uma questão importante que deve ser mencionada, e que foi muito bem lembrada por Costa (2010), é o fato de que, apesar das campanhas de triagem para a avaliação da saúde ocular das crianças serem de suma importância, elas não garantem a resolução dos problemas encontrados. Aponta o autor a não continuidade dos tratamentos posteriores como um fator sério para o seu insucesso, e menciona a existência de diversos estudos que mostram que um grande número de crianças encaminhadas ao especialista não comparece à consulta posteriormente. Nesse sentido, pode-se inferir que o professor tem um papel importante, uma vez que ele pode cobrar dos responsáveis maior comprometimento nesta questão. Além disso, há de se concordar com Moreira et al. (2001, p. 30), quando afirmam que é de suma importância “a participação da família, uma vez que a criança ir ou não ao oftalmologista depende dos pais e ou responsáveis diretos."

Em relação aos docentes, de acordo com informações obtidas na Escola Municipal Manoel Fonseca, faz parte do seu corpo docente um total de 35 professores. Quanto ao nível de escolaridade desses professores, verifica-se que 11 possuem o Curso Normal, 04 têm o terceiro grau incompleto, 15 possuem o terceiro grau completo e 05 são pós-graduados. No entanto, esta amostra foi composta por 30 professores, tendo em vista a recusa de 05 deles em participarem do estudo.

Edição Especial com os melhores trabalhos apresentados no IV ENECiências: UFF - 13 a 16 de maio de 2014. 


\section{Ensino, Saúde e Ambiente - V 7 (1), Edição Especial, maio de 2014}

As respostas dos docentes foram agrupadas, tendo-se em vista os objetivos deste estudo. De um modo geral, as respostas dos participantes do estudo ao questionário revelam que:

Questão 1 - Você concorda com a afirmação de que a escola é um importante mecanismo assistencial de promoção da equidade no atendimento das necessidades de saúde da população?

- $\quad$ Sim: $27(90,0 \%)$

- $\quad$ Não: $03(10,0 \%)$

Questão 2 - $O$ acompanhamento programado do crescimento $e$ desenvolvimento, completado por atividades e controle das doenças prevalentes na infância e na adolescência podem ser também realizadas pela escola?

- $\quad$ Sim: $29(96,7 \%)$

- $\quad$ Não: $01(3,3 \%)$

Questão 3 - Você acha que os professores da sua escola estão aptos a fazerem parte desse acompanhamento programado de prevenção, diagnóstico precoce e recuperação dos agravos à saúde?

- $\quad$ Sim: $08(26,7 \%)$

- $\quad$ Não: $19(63,3 \%)$

- $\quad$ Não responderam: $03(10,0 \%)$

Diante destas respostas, sugere-se que o desenvolvimento do Programa Saúde na Escola (PSE) ainda parece muito acanhado. Os resultados ainda não são totalmente satisfatórios, necessitando de um processo de capacitação dos dos educadores de modo a atender as diretrizes do Programa.

De acordo com Carvalho e Santana (2013, p. 8):

[...] os professores não se veem como agentes promotores de saúde [...], o que inviabiliza a transformação da escola em uma agência promotora de saúde. Entretanto, quando recebem treinamento e capacitação adequados os professores percebem a importância do papel que desempenham como educadores na promoção da saúde na escola [...]. A escola precisa, para bem desenvolver estratégias de promoção de saúde, de profissionais com boa formação, nos níveis de graduação e pós-graduação e também de conhecimento e envolvimento com a realidade local [...].

Questão 4 - O que a escola juntamente com você professor pode estar fazendo para a promoção de saúde ocular dos alunos?

- $\quad$ Comunicar à família: $08(26,7 \%)$

Edição Especial com os melhores trabalhos apresentados no IV ENECiências: UFF - 13 a 16 de maio de 2014. 


\section{Ensino, Saúde e Ambiente - V 7 (1), Edição Especial, maio de 2014}

- $\quad$ Encaminhar o aluno para profissional: $06(20,0 \%)$

- $\quad$ Realizar o teste da visão: $04(13,4 \%)$

- Observar para diagnosticar na medida do possível: 03 $(10,0 \%)$

- Realizar parceria para melhor atender os alunos: $02(6,7 \%)$

- $\quad$ Não respondeu: $02(6,7 \%)$

- Comunicar a família e encaminhar para oftalmologista: 01 $(3,3 \%)$

- $\quad$ Capacitar os professores: $01(3,3 \%)$

- $\quad$ Oferecer óculos: $01(3,3 \%)$

- Observar o aluno e incluir tema no Projeto Político Pedagógico (PPP): 01 (3,3\%)

- Observar e encaminhar para consulta: $01(3,3 \%)$

Verifica-se que a opção mais considerada pelos professores é comunicar a família e/ou encaminhar o aluno para um profissional especializado. Nesse sentido, para que isto realmente venha acontecer é necessária muita atenção por parte dos professores no sentido de identificar em sala de aula situações que possam ser decorrentes de deficiência de acuidade visual, tais como dificuldades de locomoção, de ler, copiar e queixas dos alunos.

Questão 5 - Qual a sua opinião sobre a implantação da avaliação da saúde do escolar do Programa Saúde na Escola, tendo como ponto fundamental a orientação para a saúde ocular, já que o diagnóstico dos problemas oculares em crianças por meio de oftalmologistas mostra-se muito dispendioso e mesmo inexequível em saúde pública?

- Importante, boa oportunidade e interessante: $08(26,6 \%)$

- $\quad$ Não respondeu: $06(20,0 \%)$.

- $\quad$ Ótima ideia, excelente: 06 (20,0\%).

- $\quad$ Grande ajuda, contribuirá em muito: 05 (16,7\%).

- $\quad$ Muito válido, viável e pertinente: $03(10,0 \%)$.

- $\quad$ Necessidade de urgência: 02 (6,7\%). 


\section{Ensino, Saúde e Ambiente - V 7 (1), Edição Especial, maio de 2014}

Para Laignier, Castro e Sá (2010), o enfermeiro, que hoje está inserido de forma marcante na área da saúde familiar, deve expandir seu campo de atuação para as escolas, uma vez que é um profissional competente na atuação teórica e prática e capaz de realizar grandes projetos que visam à saúde, tanto preventiva quanto curativa.

Conforme Russ, Temporini e Kara-Jose (2004), a dificuldade de deslocar o professor da sala de aula, seja para o treinamento ou para aplicação do teste de acuidade visual, é uma das dificuldades apontadas pelos profissionais em campanha de triagem. Além disso, apesar de alguns professores considerarem a triagem muito importante, são bem poucos aqueles que acham que esta é uma responsabilidade do professor, pois a maioria acha que a aplicação do teste deve ser realizada por profissionais da área da saúde, para maior exatidão. No entanto, estudos mostram que a triagem é realizada corretamente pelo professor treinado em $87,1 \%$ dos casos.

Questão 6 - Qual sua sugestão para que os professores tornem-se mais capacitados para auxiliarem nesta avaliação da saúde do escolar, principalmente no que tange a medidas preventivas de doenças visuais?

- $\quad$ Capacitação: $25(83,3 \%)$

- $\quad$ Não respondeu: $05(16,7 \%)$

Fendi et al. (2008) constaram em seus estudos a importância da participação dos professores na melhoria da saúde ocular dos alunos. Embora os dados de seus estudos mostrarem que os exames de detecção precoce de acuidade visual em escolares realizados pelos professores geralmente subestimam os valores realmente apresentados por eles, quando comparados aos valores obtidos pela avaliação dos oftalmologistas. Mesmo assim, os autores ressaltam a importância do papel do professor na identificação do aluno com problemas de visão. Todavia consideram necessária a orientação dos docentes, de forma com que possam ampliar seus conhecimentos e compreensão a respeito do papel fundamental que desempenham nas ações de saúde ocular na escola.

Russ, Temporini e Kara-Jose (2004) alegam que o contato diário e prolongado com alunos favorece a observação do estado de saúde e o desempenho visual e intelectual dos escolares pelo professor. No entanto, o professor nem sempre dispõe de conhecimentos, atitudes, habilidades e práticas no campo da saúde ocular. Havendo ainda grande descontentamento por partes dos professores responsáveis pela triagem, que aparentemente se acham despreparados para realizar tal tarefa.

Visando contribuir para melhor compreensão por parte dos professores, sobre a saúde ocular dos alunos, resolveu-se por elaborar um vídeo educativo, que pode se Edição Especial com os melhores trabalhos apresentados no IV ENECiências: UFF - 13 a 16 de maio de 2014. 


\section{Ensino, Saúde e Ambiente - V 7 (1), Edição Especial, maio de 2014}

tornar um recurso didático para uso dos docentes do Ensino Fundamental da Rede Pública da Secretaria Municipal de Educação que buscam aprimorar os seus conhecimentos em saúde ocular. O vídeo foi confeccionado por meio de contratação de profissional especializado e com recursos próprios.

A metodologia de elaboração do vídeo "De Olho na Saúde" teve como princípio a visão no processo de ensino-aprendizagem e aprender como realizar o teste de acuidade visual.

Utilizando-se de vocabulário de fácil entendimento, atentando para a importância da visão no processo de aprendizagem e do diagnóstico precoce, o vídeo apresenta 8 sequências, a saber:

Após a vinheta de abertura, ao som de melodia de cantigas de roda, tocadas em flauta, a apresentadora convida os professores do ensino fundamental a conhecerem os métodos para identificar a condição da saúde visual de seus alunos, com a aplicação de exames dentro da sala de aula. Utilizando-se de vocabulário de fácil entendimento, tenta-se realizar um diálogo com o expectador e atentar para a importância da visão no processo de aprendizagem, mostrando a importância do diagnóstico precoce, nesse processo.

Aparece uma sala de aula com alunos do ensino fundamental, aproximadamente do segundo ano. Crianças estudando e olhando para o quadro. Uma delas começa a ter dificuldade para enxergar. A professora e também apresentadora do vídeo se aproxima da criança. Conversa com ela e pergunta se está com dificuldade de enxergar a matéria. A partir daí o vídeo se desenrola em oito sequências de informações que são:
1) O que é visão;
2) anatomia do olho;
3) problemas de refração;
4) sinais e sintomas;
5) acuidade visual;
6) material utilizado;
7) procedimentos realizados;
8) critérios para encaminhamento ao oftalmologista.

A primeira sequência de aprendizagem aborda a definição de visão e a anatomia do olho, sendo denominada: "O que é visão". Apresentam-se na sequência os erros de refração para que o professor possa conhecer melhor os tipos mais comuns Edição Especial com os melhores trabalhos apresentados no IV ENECiências: UFF - 13 a 16 de maio de 2014. 


\section{Ensino, Saúde e Ambiente - V 7 (1), Edição Especial, maio de 2014}

(miopia, hipermetropia, astigmatismo e estrabismo) e incluem os principais sinais e sintomas a serem observados.

A seguir são apresentadas as ferramentas necessárias para a realização do teste, como por exemplo, a tabela de Snellen, a preparação da sala de exames e outros materiais pertinentes. Compondo a última sequência de aprendizagem, é exibido como se aplica a técnica e os critérios para encaminhamento de quem precisa de atenção especializada.

Acredita-se que este vídeo possa ser uma ferramenta útil a ser empregada pelos docentes nas ações que procuram romper com o fluxo crescente da demanda de crianças e adolescentes com déficit da acuidade visual, e que possa ampliar o acesso do escolar aos serviços de oftalmologia.

\section{CONCLUSÕES}

A pesquisa realizada demonstrou alterações oftalmológicas nos escolares e foi uma oportunidade para que vários alunos fossem avaliados quanto à sua saúde ocular. Aqueles que apresentaram algum grau de alteração foram encaminhados para consulta especializada por médico oftalmologista.

Também possibilitou evidenciar que ações de prevenção e promoção da saúde devem ser estimuladas nas escolas, onde professores e profissionais de saúde possam desenvolver atividades multidisciplinares, no intuito de corroborar para uma adequada assistência à saúde dos escolares. Mas para isso, essas ações precisam ser incorporadas à rotina dos serviços de saúde em integração com as metas da educação.

A preparação e capacitação adequada desses professores, promovida por profissionais de saúde capacitados, principalmente o enfermeiro, através da educação em saúde, poderá contribuir substancialmente com os objetivos do Programa Saúde na Escola e, em especial, com a saúde ocular da população.

A partir dessas premissas, acredita-se ser possível alcançar sucesso no combate às dificuldades de aprendizado ocasionadas pela diminuição da acuidade visual em escolares, uma vez que se considera que este seja um dos motivos responsáveis pela repetência e evasão de uma parcela considerável de crianças e adolescentes matriculados em escolas públicas. 


\section{REFERÊNCIAS BIBLIOGRÁFICAS}

CARVALHO, Denis Barros de; SANTANA Janaína Macedo. A escola promotora de saúde: o estado da arte e o mental na saúde. Disponível em:

http://www.ufpi.br/subsiteFiles/ppged/arquivos/files/VI.encontro.2010/GT.10/GT_10_0

2_2010.pdf. Acesso em: 02 mar. 2013.

COSTA, Marcio Sued. Saúde e educação na atenção primária ocular - um olhar de cidadania. 2010, 92f. Dissertação (Mestrado) - ISCTE Business School, Departamento de Gestão, Instituto Universitário de Lisboa. Lisboa-Portugal, 2010.

COUTO JUNIOR, Abelardo de Souza et al. Prevalência das ametropias e oftalmopatias em crianças pré-escolares e escolares em favelas do Alto da Boa Vista, Rio de Janeiro, Brasil. Rev. bras.oftalmol., v. 66, n.5, p. 304-308, 2007.

DANTAS, Rosane Arruda; PAGLIUCA, Lorita Marlena Freitag; ALMEIDA, Paulo César de. Validação de escala optométrica regionalizada para pré-escolares:

contribuição da enfermagem. Rev. esc. enferm. USP., v. 43, n. 2, p. 279-286, 2009.

FENDI, Lígia Issa de et al. Qualidade da avaliação da acuidade visual realizada pelos professores do programa "Olho no olho" da cidade de Marília, SP. Arq. Bras.

Oftalmol., v. 71 n. 4, São Paulo, jul./ago., 2008.

GASPARETTO, Maria Elisabete R. Freire et al. Dificuldade visual em escolares: conhecimentos e ações de professores do ensino fundamental que atuam com alunos que apresentam visão subnormal. Arq. Bras. Oftalmol., v. 67, n.1, p 65-71, fev., 2004.

GRANZOTO, José Aparecido et al. Avaliação da acuidade visual em escolares da 1a série do ensino fundamental. Arq. Bras. Oftalmol., v. 66, n. 2, p. 167-171, 2003.

LAIGNIER, Mariana Rabello; CASTRO, Marlúcia de Almeida; SÁ, Paula dos Santos Cabral de. De olhos bem abertos: investigando acuidade visual em alunos de uma escola municipal de Vitória. Esc Anna Nery Rev Enferm, v. 14, n. 1, p. 113-19, jan./mar. 2010.

MOREIRA, Thereza M. M.; PAGLIUCA, Lorita M. Freitag; ARAÙJO, Thema Leite. Refletindo sobre a saúde ocular na criança. Revista Nursing. São Paulo, v. 4, n. 33, p.30-34, fev., 2001.

RUSS, Heloisa Helena Abil; TEMPORINI, Edméa Rita; KARA-JOSE, Newton. Impacto da Campanha Olho no Olho em escolas de ensino fundamental: percepção fazer pessoal de ensino. Arq. Bras. Oftalmol., v. 67, n.2, p 311-321, 2004.

SISTON, NA; VARGAS LA. O enfermeiro na escola: práticas educativas na promoção da saúde de escolares. Enfermería Global. n. 11, nov. 2007. Disponível em: http://revistas.um.es/eglobal/article/viewFile/409/521. Acesso em: 13 fev. 2013. 